\title{
EXPLICIT SOLUTION TO CONSTRAINED LINEAR ESTIMATION
}

\author{
Xiang W. Zhuo* José A. De Doná* \\ María M. Seron* \\ * Centre for Complex Dynamic Systems and Control, \\ School of Electrical Engineering and Computer Science \\ The University of Newcastle \\ Callaghan 2308, NSW, Australia
}

\begin{abstract}
This paper investigates the structure present in constrained linear state estimation problems formulated as a quadratic optimization program subject to linear inequality constraints. Polyhedral constraints on the system disturbance, the measurement noise, and the initial state are considered. The result interprets the measurement data and prior estimate as parameters and the parameter space is partitioned into multiple regions. Within each region the state estimate can be calculated as a piece-wise affine function of the measurement data and prior estimate. The parameterized regions and coefficients of the piece-wise affine function can be precomputed offline allowing a simplified approach in implementing a moving horizon estimation scheme. Copyright ${ }^{\circledR} 2005$ IFAC.
\end{abstract}

Keywords: Constraints, Optimal Estimation, Quadratic Programming

\section{INTRODUCTION}

A state estimation problem consists of determining estimates of internal states of a dynamical system based on measured data of the dynamical system's output. The fact that the system states, in general, are not directly accessible from the measurements makes state estimation problems relevant to many areas of engineering such as communications and process control. In the case of constrained state estimation the variables are known, a priori, to satisfy various constraints. For example, states in physical systems which may be known to lie between certain limits.

A strategy to determine the optimal state estimate is to formulate the problem as a quadratic program (Goodwin et al., 2005). One of the advantages of this formulation is the simplicity of adding linear inequality constraints. However, the presence of inequality constraints precludes general recursive solutions. Thus the problem size grows without bound as more measurements are taken into account. To counteract the growing complexity, moving horizon estimation (MHE) schemes have been proposed and investigated by many authors, e.g., Muske et al. (1993), Robertson et al. (1996), Rao et al. (2001).

A common point between optimal constrained estimation and control problems is that, under suitable conditions, both problems can be formulated as quadratic programs. Recent papers have exploited the underlying geometric structure of quadratic programming to obtain explicit solutions for receding horizon control of constrained linear systems (e.g., Seron et al., 2003). The present paper applies these concepts to the framework of constrained linear state estimation in a moving horizon configuration. Polyhedral constraints on the disturbance, the measurement noise, and the initial state are considered. The result partitions the space of the measurement 
data and prior estimate into multiple regions, and within each region the state estimates can be calculated as a piece-wise affine function of the measurements and prior estimate.

The layout of the remainder of this paper is as follows. In the next section, the constrained linear estimation problem is considered and formulated as a quadratic program. In Section 3, the structure of the quadratic program subject to linear inequality constraints is explored, and an explicit solution to the problem is obtained. In Section 4, a MHE scheme using explicit solutions is considered. Finally, an example to illustrate the regions of the explicit solution is examined in Section 5 and some conclusions are presented in Section 6 .

\section{CONSTRAINED FIXED HORIZON ESTIMATION}

Consider the problem to estimate the system state $x_{N}$ given measurements $y_{1}, \cdots, y_{N}$ for the following linear time invariant, discrete system

$$
\begin{aligned}
x_{k+1} & =A x_{k}+B w_{k}, & k & =0, \cdots, N-1, \\
y_{k} & =C x_{k}+v_{k}, & k & =1, \cdots, N,
\end{aligned}
$$

where the initial state $x_{0} \in \mathbb{R}^{n}$, input disturbance $w_{k} \in \mathbb{R}^{m}$, and measurement noise $v_{k} \in \mathbb{R}^{p}$ are known to satisfy the following constraints:

$$
x_{0} \in \mathbb{X}_{0}, \quad w_{k} \in \mathbb{W}, \quad v_{k} \in \mathbb{V} .
$$

In $(2), \mathbb{X}_{0}, \mathbb{W}$ and $\mathbb{V}$ are nonempty, polyhedral sets, that is, the intersection of a finite number of closed half-spaces. In addition, assume that the disturbance sequence $\left\{w_{k}\right\}$ and the noise sequence $\left\{v_{k}\right\}$ are i.i.d. sequences having truncated Gaussian distributions

$$
p_{w}\left(w_{k}\right)=\frac{e^{-\frac{1}{2} w_{k}^{\mathrm{T}} Q^{-1} w_{k}}}{\int_{\mathbb{W}} e^{-\frac{1}{2} v^{\mathrm{T}} Q^{-1} v} d v},
$$

for $w_{k} \in \mathbb{W}$ and zero otherwise, and

$$
p_{v}\left(v_{k}\right)=\frac{e^{-\frac{1}{2} v_{k}^{\mathrm{T}} R^{-1} v_{k}}}{\int_{\mathbb{V}} e^{-\frac{1}{2} v^{\mathrm{T}} R^{-1} v} d v},
$$

for $v_{k} \in \mathbb{V}$ and zero otherwise; with $Q>0$ and $R>0$. Also, assume that $x_{0}$ is independent of $\left\{w_{k}\right\}$ and $\left\{v_{k}\right\}$ and has a truncated Gaussian distribution

$$
p_{x_{0}}\left(x_{0}\right)=\frac{e^{-\frac{1}{2}\left(x_{0}-\bar{x}_{0}\right)^{\mathrm{T}} P_{0}^{-1}\left(x_{0}-\bar{x}_{0}\right)}}{\int_{\mathbb{X}_{0}} e^{-\frac{1}{2}\left(v-\bar{x}_{0}\right)^{\mathrm{T}} P_{0}^{-1}\left(v-\bar{x}_{0}\right)} d v},
$$

for $x_{0} \in \mathbb{X}_{0}$ and zero otherwise; with $P_{0}>0$.

The constrained fixed horizon linear estimator that seeks the joint a posteriori most probable (JAPMP) estimate can be formulated as the solution to the following optimization problem:

Given a prior estimate $\bar{x}_{0}$ and measurement data $\left\{y_{1}, \cdots, y_{N}\right\}$, solve

$$
\min _{x_{0}, w_{0}, \cdots, w_{N-1}} J\left(x_{0}, w_{0}, \cdots, w_{N-1}\right),
$$

subject to (1)-(2). In (3), the objective function is defined by

$$
\begin{aligned}
& J\left(x_{0}, w_{0}, \cdots, w_{N-1}\right) \triangleq \\
& \frac{1}{2}\left\|x_{0}-\bar{x}_{0}\right\|_{P_{0}^{-1}}^{2}+\frac{1}{2} \sum_{k=0}^{N-1}\left\|w_{k}\right\|_{Q^{-1}}^{2}+\frac{1}{2} \sum_{k=1}^{N}\left\|v_{k}\right\|_{R^{-1}}^{2},
\end{aligned}
$$

where $\|x\|_{M}^{2}$ denotes $x^{\mathrm{T}} M x$ for a real vector $x$ and real symmetric matrix $M$. Further detailed discussion of the above optimization problem in connection to JAPMP state estimation can be found in Goodwin et al. (2005).

The optimization problem (1)-(4) can be expressed in a more concise form by defining the vectors

$$
\mathbf{v} \triangleq\left[\begin{array}{c}
\bar{x}_{0}-x_{0} \\
v_{1} \\
\vdots \\
v_{N}
\end{array}\right], \quad \mathbf{w} \triangleq\left[\begin{array}{c}
x_{0} \\
w_{0} \\
\vdots \\
w_{N-1}
\end{array}\right], \quad \mathbf{y} \triangleq\left[\begin{array}{c}
\bar{x}_{0} \\
y_{1} \\
\vdots \\
y_{N}
\end{array}\right]
$$

Applying the above definitions, the linear constraints defined by the system equations (1) can be written as

$$
\mathbf{y}=G \mathbf{w}+\mathbf{v}
$$

with

$$
G \triangleq\left[\begin{array}{cccc}
I & 0 & \cdots & 0 \\
C A & C B & \cdots & 0 \\
\vdots & \vdots & \ddots & \vdots \\
C A^{N} & C A^{N-1} B & \cdots & C B
\end{array}\right]
$$

Finally, using the above definitions and

$$
\begin{aligned}
& \mathbf{Q}^{-1} \triangleq \operatorname{diag}\left\{0_{n \times n}, Q^{-1}, \cdots, Q^{-1}\right\}, \\
& \mathbf{R}^{-1} \triangleq \operatorname{diag}\left\{P_{0}^{-1}, R^{-1}, \cdots, R^{-1}\right\},
\end{aligned}
$$

the objective function (4) can be rewritten as

$$
\begin{aligned}
J(\mathbf{w}) & =\frac{1}{2} \mathbf{w}^{\mathrm{T}} \mathbf{Q}^{-1} \mathbf{w}+\frac{1}{2}(\mathbf{y}-G \mathbf{w})^{\mathrm{T}} \mathbf{R}^{-1}(\mathbf{y}-G \mathbf{w}) \\
& =\frac{1}{2} \mathbf{w}^{\mathrm{T}} H \mathbf{w}+\mathbf{w}^{\mathrm{T}} F \mathbf{y}+\bar{J}
\end{aligned}
$$

where $\bar{J}$ is independent of $\mathbf{w}$, and

$$
H=\mathbf{Q}^{-1}+G^{\mathrm{T}} \mathbf{R}^{-1} G, \quad F=-G^{\mathrm{T}} \mathbf{R}^{-1} .
$$

The polyhedral constraints (2) can be expressed as a linear inequality

$$
L \mathbf{w} \leq K,
$$

where, without loss of generality, the matrix $L$ and the vector $K$ can be expressed as

$$
L=\left[\begin{array}{c}
\Phi \\
-\Phi
\end{array}\right], \quad K=\left[\begin{array}{l}
\alpha \\
\beta
\end{array}\right]+\left[\begin{array}{c}
\Lambda \\
-\Lambda
\end{array}\right] \mathbf{y} .
$$

In (9), $\Phi$ is a $q \times(N m+n)$ matrix, $\Lambda$ is a $q \times$ $(N p+n)$ matrix, $\alpha$ and $\beta$ are $q \times 1$ vectors such that $\alpha+\beta>0$, component-wise. The form (8)-(9) can be used to express $q$ interval type constraints on the components of $x_{0}, w_{k}$ and $v_{k}$. 
Utilizing the aforementioned definitions, the optimization problem defined by equations (1)-(4) is equivalent to the following quadratic program

$$
\min _{\mathbf{w}}\{J(\mathbf{w}): L \mathbf{w} \leq K\} \text {. }
$$

Let $\hat{x}_{k \mid N}$ denote the state estimate at time $k$ given the measurements $y_{1}, \cdots, y_{N}$ and let $\hat{x}_{k} \triangleq \hat{x}_{k \mid k}$. The solution to the optimization problem (10) is the vector $\hat{\mathbf{w}}^{\mathrm{T}}=\left[\hat{x}_{0 \mid N}, \hat{w}_{0 \mid N}, \cdots, \hat{w}_{N-1 \mid N}\right]$, which yields the state estimate $\hat{x}_{N}$,

$$
\hat{x}_{N}=\Gamma_{N} \hat{\mathbf{w}},
$$

where

$$
\Gamma_{N}=\left[\begin{array}{llll}
A^{N} & A^{N-1} B & \cdots & B
\end{array}\right]
$$

The constrained estimation problem (1)-(4) (and the equivalent concise formulation (10)) has been investigated by many authors (e.g., Rao et al., 2001). In practice, the problem is usually implemented in a moving horizon configuration, i.e., the fixed horizon problem is solved at each time over a window of size $N$ and the window is moved at the next time instance as a new measurement vector becomes available. The quadratic program (10) has, then, to be solved at each time instant. All of the aforementioned references propose the use of numerical optimization methods for the solution of the quadratic program. However, numerical solutions to quadratic programs can be computationally intensive and may limit the applicability to relatively slow processes. Thus, it is of interest to study the possibility of pre-computing the solution off-line so as to limit on-line computations to a minimum.

\section{EXPLICIT SOLUTION TO CONSTRAINED FIXED HORIZON ESTIMATION}

Consider the equation

$$
J(\mathbf{w})=\frac{1}{2} \mathbf{w}^{\mathrm{T}} H \mathbf{w}+\mathbf{w}^{\mathrm{T}} F \mathbf{y}+\bar{J}=c,
$$

where $\mathbf{w} \in \mathbb{R}^{N m+n}$ and $c$ is a constant. This defines an ellipsoid in $\mathbb{R}^{N m+n}$ centred at $-H^{-1} F \mathbf{y}$. The linear inequality constraint (8)-(9) defines a polyhedron in $\mathbb{R}^{N m+n}$ where the solution to the constrained optimization problem (10) must lie. The elliptical contour (12) can be transformed into a spherical contour by applying the transformation

$$
\tilde{\mathbf{w}}=H^{1 / 2} \mathbf{w},
$$

allowing the cost function (6) to be written as

$$
J(\tilde{\mathbf{w}})=\frac{1}{2} \tilde{\mathbf{w}}^{\mathrm{T}} \tilde{\mathbf{w}}+\tilde{\mathbf{w}}^{\mathrm{T}} H^{-1 / 2} F y+\bar{J} .
$$

Similarly, applying the transformation to the linear inequality constraints (8)-(9) yields

$$
\begin{aligned}
\tilde{\Phi} \tilde{\mathbf{w}} & \leq \alpha+\Lambda \mathbf{y}, \\
-\tilde{\Phi} \tilde{\mathbf{w}} & \leq \beta-\Lambda \mathbf{y},
\end{aligned}
$$

where $\tilde{\Phi}=\Phi H^{-1 / 2}$. It follows that in minimizing equation (14) subject to the linear inequality constraints (15)-(16), the optimal solution is obtained by the Euclidean projection of the unconstrained solution onto the constraint polyhedron defined by the linear inequalities (see Seron et al., 2003).

In the absence of constraints, or when the unconstrained solution lies inside the constraint polyhedron, the optimal solution to the quadratic program is the centre of the ellipsoid given by

$$
\hat{\mathbf{w}}_{\mathrm{uc}}=-H^{-1} F \mathbf{y}, \quad \tilde{\hat{\mathbf{w}}}_{\mathrm{uc}}=-H^{-1 / 2} F \mathbf{y},
$$

where the hat ^ indicates the optimal solution, and the subscript uc indicates an unconstrained solution. Observe that the second of equation (17) defines a transformation

$$
\tilde{\mathbf{w}}=-H^{-1 / 2} F \mathbf{y}
$$

between the vector space of parameters $\mathbf{y}$ and the vector space of decision variables $\tilde{\mathbf{w}}$.

In the case when the unconstrained solution lies outside the constraint polyhedron, the optimal solution lies on a hyperface of the constraint polyhedron defined by a set of active constraints of (15)-(16). For each set of active constraints there is a corresponding region in $\mathbb{R}^{N m+n}$ where the optimal solution in the transformed coordinates $\tilde{\mathbf{w}}$ can be obtained by the Euclidean projection of the unconstrained solution onto the corresponding hyperface of the constraint polyhedron.

If the following, we proceed to define the active regions corresponding to a set of active constraints. For a given matrix $M$, denote $M_{i}$, where $i$ is a set of indices, as a submatrix formed by selecting the rows of $M$ with indices in $i$. Let $q=\operatorname{rank} \Phi$, and $J \triangleq\{1,2, \cdots, q\}$. A face of the constraint polyhedron is given by the intersection with the constraint polyhedron of a hyperplane defined by a subset of $\tilde{N} \in J$ equalities within equations (15)-(16). For each face of the polyhedron, let $l \triangleq\left\{l_{1}, l_{2}, \cdots, l_{\bar{N}}: l_{k} \in J\right\}$ identify the rows within (15)-(16) such that equality holds, and let $\Delta \in \mathbb{R}^{\bar{N}}$ be a vector with elements $\Delta_{k}$ given by

$$
\Delta_{k} \triangleq \begin{cases}\alpha_{l_{k}} & \text { if } \tilde{\Phi}_{l_{k}} \tilde{\mathbf{w}}=\alpha_{l_{k}}+\Lambda_{l_{k}} \mathbf{y}, \\ -\beta_{l_{k}} & \text { if } \tilde{\Phi}_{l_{k}} \tilde{\mathbf{w}}=-\beta_{l_{k}}+\Lambda_{l_{k}} \mathbf{y}\end{cases}
$$

That is, for each face of the polyhedron, the elements of the vector $\Delta$ are the elements of vectors $\alpha$ or $\beta$ where the equality holds for the hyperplane. Combining definition (19) and equations (15)(16), a hyperplane of the constraint polyhedron is given by

$$
\tilde{\Phi}_{l} \tilde{\mathbf{w}}=\Delta+\Lambda_{l} \mathbf{y}
$$

Finally, let $s \triangleq J-l=\left\{s_{1}, s_{2}, \cdots, s_{q-\bar{N}}: s_{k} \in\right.$ $\left.J, s_{k} \notin l\right\}$ identify the constraints that are not active for each face. 
The following results define the active regions in $\mathbb{R}^{N m+n}$ for the pair $(l, \Delta)$ of active constraints and give the explicit expression of the corresponding state estimate $\hat{x}_{N}$.

Theorem 1. (Goodwin et al. (2005), § 6.5). The active region corresponding to the face characterized by the equality constraint (20) is given by

$$
\begin{array}{r}
S\left[\tilde{\Phi}_{l} \tilde{\Phi}_{l}^{\mathrm{T}}\right]^{-1}\left[\tilde{\Phi}_{l} \tilde{\mathbf{w}}-\Lambda_{l} \mathbf{y}-\Delta\right] \leq 0, \\
-\beta_{s} \leq \tilde{\Phi}_{s} \tilde{\mathbf{w}}-\Lambda_{s} \mathbf{y}-\tilde{\Phi}_{s} \tilde{\Phi}_{l}^{\mathrm{T}}\left[\tilde{\Phi}_{l} \tilde{\Phi}_{l}^{\mathrm{T}}\right]^{-1} \times \\
{\left[\tilde{\Phi}_{l} \tilde{\mathbf{w}}-\Lambda_{l} \mathbf{y}-\Delta\right] \leq \alpha_{s},}
\end{array}
$$

where $S$ is a sign diagonal matrix such that its $(k, k)$-entry is $S_{k k}=1$ if $\Delta_{k}=-\beta_{l_{k}}$ and $S_{k k}=-1$ if $\Delta_{k}=\alpha_{l_{k}}$.

Upon substituting transformation (18), the regions characterized by Theorem 1 are parameterized by the vector $\mathbf{y}=\left[\bar{x}_{0}, y_{1}, \cdots, y_{N}\right]^{\mathrm{T}}$, where $\bar{x}_{0}$ is the prior estimate and $y_{1}, \cdots, y_{N}$ are the measurements. Thus the active regions can be characterized by the data vector $\mathbf{y}$. This is formalized in the following corollary.

Corollary 2. The projection onto the parameter space $\mathbf{y}$ of the active regions defined by Theorem 1 is given by

$$
\begin{gathered}
S\left[\tilde{\Phi}_{l} \tilde{\Phi}_{l}^{\mathrm{T}}\right]^{-1}\left[-\left(\tilde{\Phi}_{l} H^{-1 / 2} F+\Lambda_{l}\right) \mathbf{y}-\Delta\right] \leq 0 \\
-\left(\tilde{\Phi}_{s} H^{-1 / 2} F+\Lambda_{s}\right) \mathbf{y}+\tilde{\Phi}_{s} \tilde{\Phi}_{l}^{\mathrm{T}}\left[\tilde{\Phi}_{l} \tilde{\Phi}_{l}^{\mathrm{T}}\right]^{-1} \times \\
{\left[\left(\tilde{\Phi}_{l} H^{-1 / 2} F+\Lambda_{l}\right) \mathbf{y}+\Delta\right] \leq \alpha_{s}} \\
\left(\tilde{\Phi}_{s} H^{-1 / 2} F+\Lambda_{s}\right) \mathbf{y}-\tilde{\Phi}_{s} \tilde{\Phi}_{l}^{\mathrm{T}}\left[\tilde{\Phi}_{l} \tilde{\Phi}_{l}^{\mathrm{T}}\right]^{-1} \times \\
{\left[\left(\tilde{\Phi}_{l} H^{-1 / 2} F+\Lambda_{l}\right) \mathbf{y}+\Delta\right] \leq \beta_{s} .}
\end{gathered}
$$

Having defined the active regions, the constrained solution to the optimization problem can be obtained by the following theorem.

Theorem 3. For each active region defined by equations (23)-(25), the optimal state estimate $\hat{x}_{N}$ is given by

$$
\hat{x}_{N}=M_{l} \mathbf{y}+b_{l},
$$

where

$$
\begin{gathered}
M_{l}=\Gamma_{N} H^{-1 / 2}\left(\tilde{\Phi}_{l}^{\mathrm{T}}\left[\tilde{\Phi}_{l} \tilde{\Phi}_{l}^{\mathrm{T}}\right]^{-1} \Lambda_{l}-H^{-1 / 2} F\right. \\
\left.+\tilde{\Phi}_{l}^{\mathrm{T}}\left[\tilde{\Phi}_{l} \tilde{\Phi}_{l}^{\mathrm{T}}\right]^{-1} \tilde{\Phi}_{l} H^{-1 / 2} F\right), \\
b_{l}=\Gamma_{N} H^{-1 / 2} \tilde{\Phi}_{l}^{\mathrm{T}}\left[\tilde{\Phi}_{l} \tilde{\Phi}_{l}^{\mathrm{T}}\right]^{-1} \Delta .
\end{gathered}
$$

PROOF. The result follows from Goodwin et al. (2005) Theorem 6.5.2 with the state estimate given by (11).
This result allows the state estimate $\hat{x}_{N}$ to be computed as a piece-wise affine function of the vector $\mathbf{y}$, where the coefficients of the affine functions can be precomputed offline.

\section{MOVING HORIZON IMPLEMENTATION}

In practice, the fixed horizon problem (1)-(4) is usually implemented in a moving horizon sense, that is, at each time instance $k$, problem (1)-(4) is solved over a window of size $N$ that is "moved" forward as new measurements become available. An important component in moving horizon estimation is the incorporation of an arrival cost. It has been shown in Rao et al. (2001) that this element is pivotal to the stability and performance of the estimator. In the case of the objective function of the form defined by equation (4), the arrival $\operatorname{cost} \theta\left(x_{k-N}\right)$ is the first term on the right hand side of (4)

$$
\theta\left(x_{k-N}\right) \triangleq \frac{1}{2}\left\|x_{k-N}-\hat{x}_{k-N}\right\|_{P_{k-N}^{-1}}^{2},
$$

where $\hat{x}_{k-N}$ is the moving horizon state estimate obtained at time $k-N$, and $P_{k-N}$ is a qualitative measure of that state estimate. For the fixed horizon problem (1)-(4), $k$ in equation (29) equals $N$, and $\hat{x}_{0}=\bar{x}_{0}$.

Several possibilities of including an arrival cost for constrained linear systems have been examined by Rao et al. (2001). In particular, using (29) as the arrival cost and letting $P_{k-N}$ be equal to the steady-state solution of the Riccati equation associated with the Kalman filter leads to an estimator known as the steady-state MHE.

The steady state MHE for a horizon length $N$ solves, at time $k$,

$$
\min _{x_{k-N}, w_{k-N}, \cdots, w_{k-1}} \phi\left(x_{k-N}, w_{k-N}, \cdots, w_{k-1}\right)
$$

where

$$
\begin{aligned}
& \phi\left(x_{k-N}, w_{k-N}, \cdots, w_{k-1}\right)= \\
& \frac{1}{2} \sum_{j=k-N}^{k-1}\left\|w_{j}\right\|_{Q^{-1}}^{2}+\frac{1}{2} \sum_{j=k-N+1}^{k}\left\|v_{j}\right\|_{R^{-1}}^{2}+\theta\left(x_{k-N}\right),
\end{aligned}
$$

subject to (1) and (2). In the case of a linear time-invariant system, the solution to the MHE problem (30)-(31) is identical to the fixed horizon problem (3)-(4). (For the fixed horizon problem (3)-(4), time 0 must be interpreted as the current time $k$ of the moving horizon implementation.)

Implementing the moving horizon estimator described above requires $N$ past estimates to be stored. Each of these stored estimates is used $N$ steps ahead. Namely, the stored past estimate $\hat{x}_{k-N}$ is propagated to the current estimate window at time $k$ as part of the arrival cost (29). Upon 


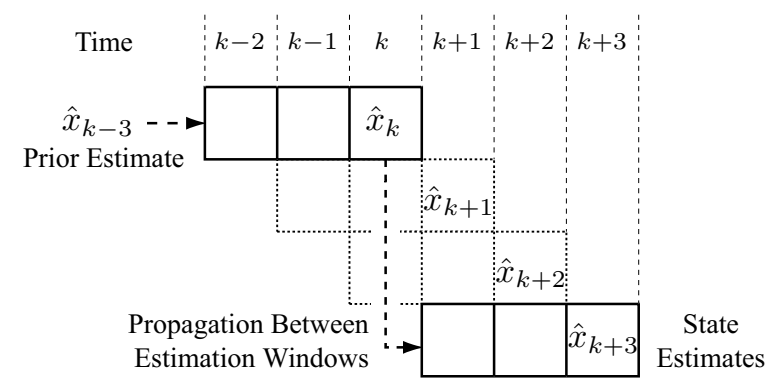

Fig. 1. MHE scheme for horizon $N=3$.

obtaining the current state estimate $\hat{x}_{k}$, this new estimate is to be stored and used at a future time, as the estimation window moves to time $k+N$, to obtain the estimate $\hat{x}_{k+N}$. This is a common method of propagating the state estimate between estimation windows. An illustration of this idea for a MHE scheme with horizon $N=3$ is depicted in Figure 1.

At each time instance $k$, the solution to the steady-state MHE can be obtained using the explicit solution. Problem (30)-(31) is parameterized by the vector $\mathbf{y}=\left[\hat{x}_{k-N}, y_{k-N+1}, \cdots, y_{k}\right]^{\mathrm{T}} \in$ $\mathbb{R}^{N p+n}$ (cf. (5)-(6)) and the corresponding active regions are defined in Corollary 2. Since the linear system dynamics (1) and polyhedral constraints (2) are time invariant, it follows that the regions are also time invariant allowing them to be computed a priori and used repeatedly. Similarly, the coefficients of the piece-wise affine function that gives the state estimate for every region are time invariant and need to be calculated once only.

The solution to problem (30) can thus be obtained by first finding, for a given data vector y (measurements and previous estimate), the region for which the vector y satisfies equations (23)-(25) in Corollary 2. Upon finding the active region, the coefficients of the piece-wise affine function (26) are uniquely determined and the state estimate $\hat{x}_{k}$ is obtained with a simple calculation.

\section{SIMULATION EXAMPLE}

We now present a simple example to illustrate the parameter space partition of an explicit solution.

Example 5.1. Consider a constrained estimation problem of the form (1)-(4) where the linear system is given by

$$
\begin{gathered}
A=\left[\begin{array}{cc}
1.589 & -0.771 \\
1 & 0
\end{array}\right], \quad B=\left[\begin{array}{c}
0.5 \\
0
\end{array}\right], \\
C=\left[\begin{array}{ll}
0.222 & 0.201
\end{array}\right] .
\end{gathered}
$$

We consider a truncated Gaussian distribution on the disturbance sequence $\left\{w_{k}\right\}$, that is, the distribution is a scaled Gaussian distribution $N(0, Q)$ in the region $[-1,1]$ and zero elsewhere. The system state $x_{0}$ and the noise sequence $\left\{v_{k}\right\}$ are assumed to be unconstrained. We take $Q=8$, $R=0.1$, and $P_{0}$ is obtained from the steady state error covariance of the Kalman Filter. The truncated Gaussian distribution imposes a linear inequality constraint of the form (9) with $\Phi=$ $\left[\begin{array}{ll}0_{N m \times n} & I_{N m \times N m}\end{array}\right], \alpha=\beta=(1, \cdots, 1)^{\mathrm{T}}$, and $\Lambda=0_{N m \times N m+n}$.

The estimation problem is formulated in a moving horizon configuration with horizon length $N=3$. To initialize the estimator, the first three initial state estimates $\hat{x}_{j}, 0 \leq j \leq 3$ are set equal to the true system states. For the simulation, the initial system state $x_{0}=\left[\begin{array}{ll}10 & -10\end{array}\right]^{\mathrm{T}}$ was set to achieve an exponentially decaying state evolution. At time instance $k$, the prior estimate $\bar{x}_{0}$ in the quadratic objective function (4) is taken as the state estimate from the previous horizon window, that is, $\bar{x}_{0}=\hat{x}_{k-N \mid k-N}$. The resulting estimator is the steady-state MHE.

The piece-wise affine solution given in Section 3 was computed for the above estimation problem. Recall that the regions are parameterized at time $k$ by the vector $\mathbf{y}=\left[\hat{x}_{k-N}, y_{k-N+1}, \cdots, y_{k}\right]^{\mathrm{T}}$. To be able to illustrate the partitions in two dimensions, the state space partition, shown in Figure 2, was obtained by projecting the active regions onto the space of the vector $\hat{x}_{k-N}$ and letting $y_{i}, k-N+1 \leq i \leq k$, be equal to zero. The first state estimate shown in the figure, where the prior estimate was obtained from a MHE, is $\hat{x}_{6}$. (Estimates before $\hat{x}_{6}$ are obtained with a priori estimates that are, in this case, equal to the actual system states.) State estimate $\hat{x}_{6}$ and the successive estimates are plotted in Figure 2 along with the actual states for comparison.

Finally, to see how the regions are affected by the horizon length and the measurement noise vari-

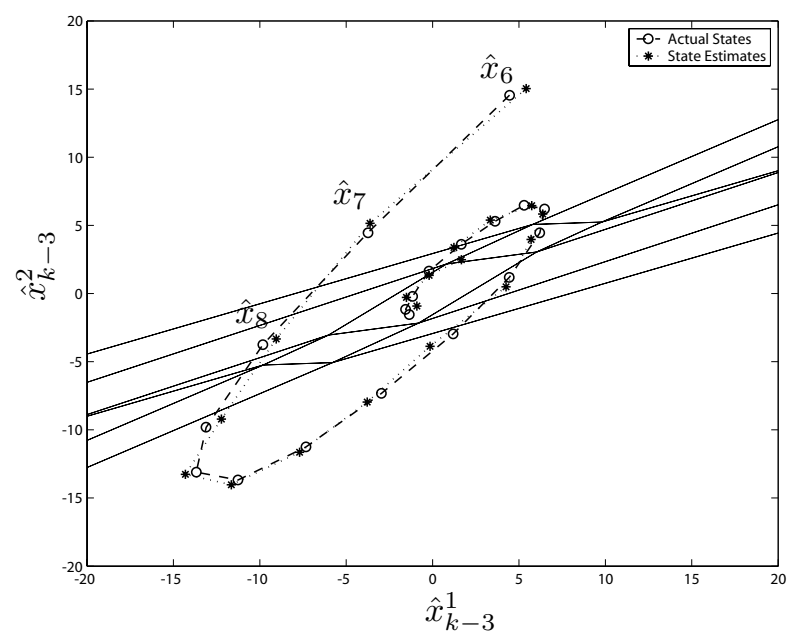

Fig. 2. Moving horizon state estimates $\hat{x}_{6}, \hat{x}_{7}$, $\hat{x}_{8}, \cdots$, in the state space partition for Example 5.1 with horizon length $N=3$ (cut corresponding to $y_{k-2}=y_{k-1}=y_{k}=0$ ). 

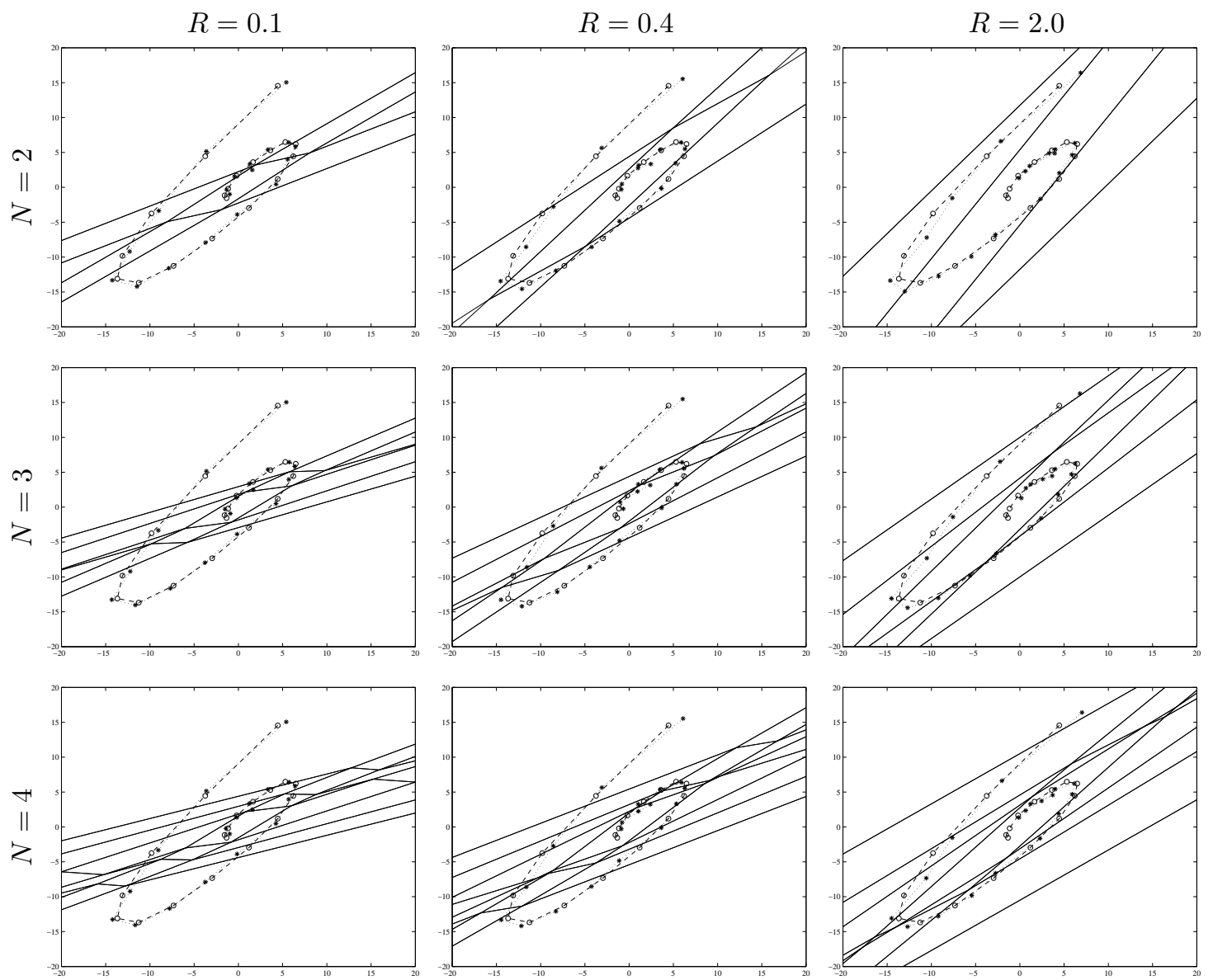

Fig. 3. State space partition for combinations of $R=0.1,0.4,2.0$ and $N=2,3,4$ for the system described in Example 5.1 (cuts corresponding to $y_{k-N+1}=y_{k-N+2}=\cdots=y_{k}=0$ ).

ance, successive values of horizon length $N=2$, $N=3$ and $N=4$ in combination with $R=0.1$, $R=0.4, R=2.0$ were used in the objective function (4). The resulting regions corresponding to the combinations of $N$ and $R$ are shown in Figure 3. Observe in the figure that the unconstrained (central) regions increase in size as the measurement noise variance $R$ increases. Lastly, the increase in the horizon length $N$ generates additional regions in the parameter-space partition.

\section{CONCLUSIONS}

We have shown that an explicit solution can be obtained for a fixed horizon constrained linear estimation problem formulated as a quadratic program. The optimal JAPMP state estimator can thus be precomputed as a piece-wise affine function of the measurement data and prior state estimate. The proposed explicit solution can then be used to simplify the online calculations required in the moving horizon implementation of the state estimator, which constituted one of the motivations of the present study. Another motivation for the explicit solution is to get a better understanding of the structure of the state estimator and the influence of different design (tuning) parameters.

\section{REFERENCES}

Goodwin, G.C., M.M. Seron and J.A. De Doná (2005). Constrained Control and Estimation. An Optimisation Approach. Springer.

Muske, K.R., J.B. Rawlings and J.H. Lee (1993). Receding horizon recursive state estimation. American Control Conference pp. 900-904.

Rao, C.V., J.B. Rawlings and J.H. Lee (2001). Constrained linear estimation — a moving horizon approach. Automatica 37, 1619-1628.

Robertson, D.G., J.H. Lee and J.B. Rawlings (1996). Moving horizon-based approach for least-squares estimation. AIChE Journal 42(8), 2209-2224.

Seron, M.M., G.C. Goodwin and J.A. De Doná (2003). Characterization of receding horizon control for constrained linear systems. Asian Journal of Control 5, 271-286. 